\begin{tabular}{|c|c|c|c|c|}
\hline & Bullous Impetigo & Dermatitis herpetiformis & Bullous pemphigoid & Linear iga dermatosis \\
\hline & & Pruritic papules & & \\
\hline & & and vesicles on the extensor surfaces & Tense vesicles & \\
\hline & Blisters of smooth walled content & of the limbs, & or bullae on erythematous base on the inner & \\
\hline \multirow[t]{2}{*}{ Clinical Features } & $\begin{array}{l}\text { initially clear and later } \\
\text { turbid }\end{array}$ & $\begin{array}{l}\text { buttocks, shoulders, nape of } \\
\text { neck, scalp }\end{array}$ & $\begin{array}{l}\text { surface of the thighs, forearms, axillary folds, } \\
\text { palms, soles }\end{array}$ & $\begin{array}{l}\text { Vesicles or bullae on perigenital area, } \\
\text { extremities, trunk, face }\end{array}$ \\
\hline & Staphylococcus aureus & & & Autoinmune, drugs induced, infections, \\
\hline \multirow[t]{2}{*}{ Aetiology } & epidermolytic toxin & Inmunogenetic & Autoinmune & trauma and burns \\
\hline & & & & $\begin{array}{l}\text { Subepidermal cavity with neutrophils along the } \\
\text { basement membrane }\end{array}$ \\
\hline & Vesicular subcorneal pustules with & Subepidermal cavity with neutrophils & Subepidermal cavity with an inflammatory & vacuolar degeneration, eosinophils \\
\hline \multirow[t]{3}{*}{ Histology } & accumulations of neutrophils & in the dermal papillae & infiltrate, predominantly of eosinophils & may be present \\
\hline & & & & LAD-1 \\
\hline & & & & BP230 \\
\hline \multirow[t]{2}{*}{ Antigen } & None & Transglutaminase & $230 \mathrm{kDa}, 180 \mathrm{kDa}$ & 97-kDa, 120-kDa antigen \\
\hline & & & Linear deposition of C3 and IgG along the & Linear deposition of IgA along the basement \\
\hline \multirow[t]{3}{*}{ Dif } & None & Granular deposition of IgA & basement membrane & membrane \\
\hline & & & & -Oral dapsone \\
\hline & & & & -Oral dapsone + oral steroids \\
\hline Treatment & Topical and systemic antibiotic & Gluten free diet, oral dapsone & Topical and oral steroids. Oral dapsone & -Colchicine and intravenous immunoglobulins \\
\hline
\end{tabular}

Background and aims We studied the feto-placental interface, in Pregnancy Induced Hypertension (PIH), to present his specific structural modifications.

Method We have studied the microscopical modifications of 68 placentas obtained after delivery for two equal groups representing mothers with PIH and normotensive. The samples obtained by sections were specifically prepared for the study using three types of histological stains. We used optical microscopy for observing mainly the lumen of spiral arteriole and changes in its intimate and medial tunica.

Results We registered the following specific structural modifications in the pregnancies with PIH : fibrosis in the middle of the villosity, fibrinoid necrosis, condensation of stromal connective tissue, syncytial layer agglutinations of the villous or intervillous spaces (nodes, buds, or bridges), thrombosis and/or infarction of the spiral vessels and villous capillary endothelial atheromatosis. Conclusions Our study was done to find a better understanding of the histo-logical changes of the preeclamptic feto-maternal interface concerning his role in PIH. The morphological modifications of the feto-placental interface in the PIH represent a marker of the fetal and postnatal hypoxia/ischemia with an immediate and late impact upon their cerebral development.

\section{PO-0365 HISTOLOGICAL ASPECTS OF THE FETO-PLACENTAL INTERFACE IN THE GESTATIONAL DIABETES MELLITUS}

${ }^{1} \mathrm{R}$ llie, ${ }^{2} \mathrm{C}$ llie, ${ }^{2} \mathrm{~F}$ Capitan, ${ }^{2} \mathrm{~A}$ Nyiredi, ${ }^{2}$ Enatescu. ${ }^{1}$ Pathology, Emergency Children's Hospital Louis Turcanu, Timisoara, Romania; ${ }^{2}$ Neonatology, Victor Babes University of Medicine and Pharmacy, Timisoara, Romania

\subsection{6/archdischild-2014-307384.1013}

Background and aims We studied the feto-placental interface, in the Gestational diabetes mellitus (GDM), to present his specific structural modifications and his cellular injuries.

Method An optical microscopic analysis was performed on 30 placentas, obtained after delivery for two equal groups representing mothers with GDM and normal pregnancies. The samples obtained by sections were specifically prepared for the study using three types of histological stains. The histological observation centred upon the: trophoblast, villous stroma and fetal capillary. The statistical study of the data was performed using SPSS 17.0.

Results Through optical microscopy were identified varying degrees of lesions consisting of: villous oedema, proliferation and villous fibrosis of the capillaries, large number of syncytial knots, important fibrinoid necrosis, moderate fibrin thrombi, hyperplasia of the syncytiotrophoblast, chorangiosis, slightly thickened of the basement membrane of the feto-maternal interface.

Conclusion Histological changes in the placentas of women with GDM are significant factors contributing to fetal anoxia with impact on placental vascular permeability. A diabetic milieu causes vascular dysfunction, increasing angiogenesis in GDM is considerred to be the cause of the placental abnormalities and complications (miscarriage, stillbirth, macrosomia, and congenital anomalies).

\section{P0-0366 PRADER-WILLI SYNDROME: 3 CASES STUDIES}

F Kamoun, TH Kamoun, L Sfaihi, K Baklouti, I Maaloul, I Chabchoub, M Hachicha. Pediatrics Department, Hedi Chaker Hospital, Sfax, Tunisia

\subsection{6/archdischild-2014-307384.1014}

Backgroud and aims Prader-Willi syndrome is a highly variable genetic disorder affecting multiple body systems.

Aims Study the clinical diagnostic criteria and genetic testing confirmation of Prader-Willi syndrome.

Methods We report 3 cases of Prader-Willi syndrome over a period of 7 years between 2007 and 2013.

Results It's about 3 boys. The age of diagnosis was 15 days (first patient), 8 months (second patient) and 6 years (third patient). History of hypotonia with poor suck was found in 2 cases and recurrent respiratory tract infection in 1 case. 
Diagnostic was suspected on hypotonia with poor suck in the neonatal period in the first case, hypotonia with history of feeding difficulty and psychomotor developmental delay in the second case and hyperphagia with obesity in the third case.

Physical exam showed facial dysmorphy in 1 case, bilateral cryptorchidism in the 3 cases and obesity $(\mathrm{BMI}=34.3)$ in the third case.

Chromosome analysis with fluorescence in situ hybridization (FISH) confirmed the diagnosis with identification of the deletion $15 \mathrm{q} 11.2-\mathrm{q} 13$ in the three cases.

The average retreat was 2 years; the evolution was marked by morbid obesity $(\mathrm{BMI}=57)$ with hypertension and psychiatric disturbance with hyperactivity in the third case and significant weight gain at the age of 10 months in the second case.

Conclusion Prader Willi must be suspected in all newborns with unexplained persistent hypotonia and confirmed by chromosome analysis. Early diagnosis is important to effective long-term management.

\section{PO-0367 FORMULA INTOLERANCE AND ORTHODOX DIETARY LAWS - A PASSOVER CONNEXION}

K Kaufman. Psychiatry and Neurology, Rutgers Robert Wood Johnson Medical School, New Brunswick, USA

\subsection{6/archdischild-2014-307384.1015}

Background When neonates and infants are not breastfed or require supplemental feedings, American Academy of Paediatrics recommends iron-supplemented formula. Cow's milk-based formula can be allergenic; however, this allergic response may be secondary to formula components as opposed to milk. The allergen may be inscrutable leading to false assumptions. In this case, an unusual religious clue solved such mystery.

Method Case analysis

Results An orthodox Jewish infant initially breastfed was transitioned to formula. Enfamil 4ounces q4hours caused upper respiratory mucus build-up directly post 'bottle-time'; formula was changed to ProSobee (soy-based) with resolution of 'formula intolerance.' At 12 months, cow's milk was initiated without allergic response. At 15 months, when diet included solid foods, she developed a peri-oral rash temporally associated with meals in her parents' home and following a meal in another family's home. Initial assessment revealed no new foods during these meals; however, this new allergy occurred during Passover, with strict dietary laws, suggesting a common new food. Ashkenazi Passover dietary laws preclude kitniyot (legumes, derivatives and associated crops including corn/soybeans/corn oil/soybean oil). Common Passover cooking oils include safflower oil. As both families were Ashkenazi Jews, the apparent common new food was safflower oil used in both homes. Safflower oil is an Enfamil ingredient. The presumptive allergen common to formula and Passover meals was safflower oil.

Conclusions Allergen determination may be secondary to fortuitous double exposure. Complete clinical histories need to allow for possible double exposure with willingness to consider cultural, religious, dietary law, and holiday dietary law factors.

\section{PO-0368 WITHDRAWN}

\section{PO-0369 A NIEMANN-PICK DISEASE TYPE C (NP-C) SUSPICION INDEX TOOL TO AID DIAGNOSIS IN PAEDIATRIC PATIENTS}

${ }^{1} \mathrm{CM}$ Lourenco, ${ }^{2} \mathrm{M}$ Pineda, ${ }^{3} \mathrm{E}$ Mengel, ${ }^{4} \mathrm{~B}$ Heron, ${ }^{5} \mathrm{I}$ Imrie, ${ }^{6} \mathrm{SA}$ Jones, ${ }^{7} \mathrm{~V}$ van der Linden, ${ }^{8 \mathrm{H}}$ Jahnova, ${ }^{9} \mathrm{P}$ Jesina, ${ }^{10} \mathrm{P}$ Karimzadeh, ${ }^{11} \mathrm{~V}$ Valayannopoulos, ${ }^{12} \mathrm{JM}$ Torres, ${ }^{13} \mathrm{~S}$ Kolb. ${ }^{1}$ Clinical Genetics, University of Sao Paulo, São Paulo, Brazil; ${ }^{2}$ Fundación Hospital Sant Joan de Deu CIBERER, Instituto de Salud Carlos III, Barcelona, Spain; ${ }^{3} V i l l a$ Metabolica, ZKJM MC University Mainz, Mainz, Germany; ${ }^{4}$ Centre Référence Des Maladies Lysosomales, CHU Trousseau APHP, Paris, France; ${ }^{5}$ Central Manchester and Manchester Children's Foundation Trust, University of Manchester, Manchester, UK; ${ }^{6}$ Manchester Centre for Genomic Medicine, Central Manchester Universty Hospitals Foundation Trust, Manchester, UK; ${ }^{7}$ Paediatrician, Association for Assistance to Disabled Children (AACD), Pernambuco, Brazil; ${ }^{8}$ Institute of Inherited Metabolic Disorders, Charles University, Praque, Czech Republic; ${ }^{9}$ Department of Pediatrics and Adolescent Medicine, Charles University, Prague, Czech Republic; ${ }^{10}$ Pediatric Neurology Research Center, Shahid Beheshti University of Medical Sciences, Tehran, Iran; ${ }^{11}$ Centre Référence Des Maladies Héréditaires Du Métabolisme de l'Enfant Et de l'Adulte, Hôpital Universitaire NeckerEnfants Malades, Paris, France; ${ }^{12}$ Biostatistics, Syntax for Science SL, Basel, Switzerland; ${ }^{13}$ Global Medical Lead, Actelion Pharmaceuticals Ltd, Allschwil, Switzerland

\subsection{6/archdischild-2014-307384.1016}

NP-C is a lysosomal lipid storage disorder caused by mutations in NPC1 or NPC2 genes. NP-C can present with a range of visceral, neurological and psychiatric symptoms that vary with age. A suspicion index (SI) tool was developed to assist clinicians achieve early diagnosis. The tool accurately predicts NP-C in patients $>4$ years of age but performs poorly in paediatric $(\leq 4$ years) patients. The present study aimed to utilise the characteristic symptomatology of NP-C in paediatric patients to develop a novel tool to assist paediatricians to identify patients for NP-C testing.

Paediatric patients were classified according to diagnosis: NP-C suspected and confirmed $(n=106)$; NP-C suspected but negative ( $\mathrm{n}=31$ ); control (no suspicion of NP-C; $\mathrm{n}=63$ ). Symptomatology data were collected retrospectively by questionnaire and summarised descriptively. The relationships between individual symptoms and likelihood of confirmed diagnosis of NP-C were defined by statistical modelling. The final tool was developed iteratively using combinations of symptoms until optimal discriminatory power was achieved.

The characteristic symptomatology of paediatric NP-C patients was identified; visceral symptoms were more prominent compared with older patients. The new tool discriminates well between NP-C confirmed, NP-C negative and control subjects. Statistical analysis demonstrates superior sensitivity and specificity of the paediatric tool compared to the original tool. The newly developed paediatric NP-C SI tool will help paediatricians to identify more paediatric patients with a high suspicion of NP-C, leading to more referrals for specialist testing thus improving early diagnosis and management of NPC-disease in paediatric patients.

Supported by Actelion Pharmaceuticals Ltd.

\section{PO-0370 GERM CELLS INDUCED FROM HUMAN UMBILICAL CORD MESENCHYMAL CELL-DERIVED INDUCED PLURIPOTENT STEM CELLS BY BMP4}

${ }^{1} \mathrm{~L} \mathrm{Ma},{ }^{2} \mathrm{~T}$ Wang, ${ }^{3} \mathrm{YB}$ Chen. ${ }^{1}$ Pediatrics, The Second Affiliated Hospital of Shantou University Medical College, Shantou, China; ${ }^{2}$ Pediatrics, Capital Institue of Pediatrics, Beijing, China; ${ }^{3}$ Pediatrics, Guangdong Maternity and Child Health Care Hospital, Guangzhou, China

10.1136/archdischild-2014-307384.1017 